This item was submitted to Loughborough's Research Repository by the author.

Items in Figshare are protected by copyright, with all rights reserved, unless otherwise indicated.

\title{
Preperformance routines
}

PLEASE CITE THE PUBLISHED VERSION

http://dx.doi.org/10.4135/9781483332222.n222

PUBLISHER

(c) SAGE Publications

VERSION

AM (Accepted Manuscript)

LICENCE

CC BY-NC-ND 4.0

REPOSITORY RECORD

Jackson, Robin C.. 2019. "Preperformance Routines". figshare. https://hdl.handle.net/2134/35514. 
Jackson, R. C. (2014). Pre-performance routines. In R. C. Eklund \& G. Tenenbaum (Eds.) Encyclopedia of sport and exercise psychology. Sage Publications.

\section{PRE-PERFORMANCE ROUTINES}

Pre-performance routines refer to the consistent sequence of thoughts and actions in which a performer engages before executing a skill. Typically, they are associated with performing selfpaced skills in which the performer decides when to initiate the action. Examples include sports such as golf, snooker, archery and static target shooting, as well as individual elements of team sports, such as the basketball free-throw, goal kicks in rugby and soccer penalty kicks. Research in the area has addressed both physical (i.e., actions such as taking deep breaths and looking towards the target) and mental (i.e., thoughts or cue words such as "focus") components of routines. Researchers have also looked to make inferences about mental states from observing physical features such as routine consistency, or from other physiological or behavioral measures. Routines may contain ritualized components or superstitious thoughts and behaviors that, arguably, have a different origin.

\section{Routine Consistency}

When observing pre-performance routines a defining feature is their behavioral consistency. For example, in studies examining routine times, high-skilled basketball free-throw shooters, golf putters, and rugby goal kickers have a dominant behavioral pattern that is followed before each attempt. This means that their routine times and the behaviors exhibited are more consistent relative to low-skilled or less-experienced performers. Some researchers have hypothesized that routine consistency facilitates higher levels of performance but the evidence 
for this is somewhat mixed. On the one hand, there is some evidence that routine consistency is positively correlated with performance. For example, high-skilled golfers have been found to use their dominant pre-putt routine more frequently than beginner golfers and routine consistency is correlated with golfers' handicap. In college basketball, routine time consistency has been found to correlate with free-throw success, with more successful players again displaying more consistent times. Further, professional basketball players have been found to be less successful when deviating from their dominant behavioral sequence, most notably when performers added something to their routine (e.g., taking a deep breath). Related to this, it has also been shown that disrupting the rhythm of the routine harms performance. Specifically, speeding up or slowing down basketball players' free-throw routines did not impair performance whereas disrupting the relative timing of different components (e.g., speeding up one aspect, slowing down another) led to lower shooting accuracy.

A problem with inferring from this that routine consistency causes good (or improved) performance is that showing two things are related to each other does not allow us to say that one causes the other. In addition, it is notable that there is often considerable variability between different performers both in terms of the time and content of their routines. Looking at routine time, there has been no advantage found for longer or shorter routines and large individual differences are apparent in some skills. For example, in one study of World Cup rugby union goal kicks, goal kickers were shown to have concentration times ranging from four seconds to more than 20 seconds. Similarly, research into basketball free-throws in the NBA play-offs has shown that there is no difference in success rates between routines of brief, regular, and long durations. Crucially, in the few studies where researchers have attempted to improve routine consistency, they have failed to bring about meaningful or lasting improvements in performance. 
As some researchers have noted, the behavioral consistency we see in expert performers might be a by-product of the hundreds or thousands of hours of repetitive practice.

\section{Content and Function of Mental Routines}

Researchers have hypothesized that the mental and physical components of routines have at least three possible functions. First, they can focus performers' attention, helping them to concentrate on the relevant aspects of the task and block out distractions. The nature of the distractions might be external to the performer, such as crowds making noise or waving their arms, or might be internal in the form of distracting thoughts such as "what if I miss?" Second, (and closely related to internal distraction) the mental aspects of a routine can help performers regulate their thoughts, behavior, and emotions. Researchers have suggested this is a key function of routines because a defining feature of self-paced skills is that performers have much more time to think and reflect than in externally-paced, reactive skills. For example, tennis players have a split-second to react to a passing shot during a rally, but have up to 25 seconds between points in which to consider the situation, the score, or their emotional state. In a broader sense then, routines are the vehicle through which performers try to deploy tools to help regulate their emotional, behavioral, and cognitive responses to competitive situations. Last, a consistent sequence of thoughts and behaviors can help trigger or cue automatic, fluent performance and prevent performers from trying to consciously control their actions. This links to the large body of research indicating that that anxiety can lead skilled performers to try to consciously control their actions, resulting in 'choking'. 
The specific function of any individual routine will be reflected in the nature of the content and there is evidence that performers use mental routines for different purposes. For example, research has shown that high-skilled golfers collectively employ a range of psychological 'tools' in their pre-shot routines, including distraction techniques, relaxation strategies, cue words, positive self-talk and imagery. These psychological 'tools' align with the aforementioned functions and are consistent with the '5-step strategy' proposed by Robert Singer and colleagues, a preparation strategy for performing self-paced skills (e.g., basketball free throw) that has received good empirical support. The steps consist of 'Readying' (preparing for the act), 'Imaging' (visualizing the movement), 'Focusing' (on a meaningful external cue, e.g., the rim of the basketball hoop), 'Executing' (with a quiet mind, allowing the movement to flow automatically) and 'Evaluating' (a brief assessment of the effectiveness of each of the previous steps once the skill has been performed). The readying and imaging steps are directed towards self-regulation and enhancing self-efficacy. The focusing and executing steps are clearly directed towards attentional processes. The focusing stage encourages a task-relevant, external focus, while the executing step is an attempt to prompt automatic, effortless performance that is free of conscious interference (e.g., over-analyzing movement technique).

Most experimental research into the mental function of routines has been focused on what performers attend to just before executing the skill. Researchers have used various techniques, from directly manipulating this state through verbal instructions to drawing inferences from physiological data, such as heart rate or measures of brain activity. Using direct instructions, there is a significant body of research supporting the efficacy of an external, compared to an internal, focus of attention, which involves focusing on the effects of movements rather than the movements themselves. The benefits are evident both in learning new skills and 
executing well-learned skills. For example, in one study skilled golfers performed better when attending to the motion of the club head than when attending to the motion of their arms as they performed 'chip' shots to a target. In interpreting findings from this type of study, researchers have pointed to the self-organizing nature of the motor system, arguing that an external focus facilitates this whereas an internal focus constrains the system, making it less efficient.

Consistent with this work, many performers incorporate a specific external visual focus as part of their mental routine. For example, rugby player Jonny Wilkinson uses the word 'Spot' to prompt him to focus on the very specific point on the ball he wants to strike when taking kicks at goal.

Psychophysiological studies mostly have been focused on heart rate, electroencephalogram (EEG), and visual gaze data to compare what expert and less-skilled performers are attending to during their routines. EEG studies record different types of brain activity (e.g., alpha, beta) by placing surface electrodes on different parts of the scalp. Analysis of activity in the few second before skill execution has commonly been interpreted as reflecting an acquired ability to suppress conscious control processes. This is evidenced by increased left hemisphere alpha activity, a change in the ratio of activity in the left and right hemisphere, a more widespread decrease in cerebral activity, or lower coherence between left hemisphere and frontal midline activity in the few seconds prior to skill execution. Results from research using brain imaging techniques such as fMRI to compare activity in expert and novice golfers as they imagine their pre-shot routines are consistent with this interpretation. Thus, overall brain activation is lower for experts and appears more efficiently organized compared to diffuse brain activity seen in novices.

Research using heart rate has focused on deceleration in the few seconds before skill execution. Heart rate deceleration has been shown to be associated with an external focus of 
attention and decreased cortical activity whereas greater cortical activity and an internal focus of attention is associated with heart rate acceleration. This research has corroborated findings using other methods (e.g., verbal instructions to manipulate attention). For example, there is evidence that heart rate deceleration is more pronounced in high-skilled than less-skilled golfers. Adding further weight to these findings, researchers have also monitored where performers look during their routines, notably on the 'quiet eye' period, that is, the final fixation (or tracking of a moving target) the performer makes on a location or object before initiating movement. Amongst other things, this research has shown that experts tend to have longer quiet eye duration and, what is more, that quiet eye training improves performance. The quiet eye period has also been shown to relate to electrocortical activity indicating a motor programming or preparatory function.

\section{Conclusion}

Pre-performance routines contain behavioral and mental components through which performers attempt to regulate their thoughts, emotions and actions. In so doing, routines direct performers' attention, help control psychological and physiological responses to stress, and allow motor processes to run-off with minimal conscious interferences. The weight of research indicates that it is these functions, rather than routine time or consistency per se, that facilitate performance.

Robin C. Jackson

\section{FURTHER READINGS}

Bertollo, M., Saltarelli, B., \& Robazza, C. (2009). Mental preparation strategies of elite modern pentathletes. Psychology of Sport and Exercise, 10, 244-254. doi:

10.1016/j.psychsport.2008.09.003 
Cotterill, S. T., Sanders, R., \& Collins, D. (2010). Developing effective pre-performance routines in golf: why don't we ask the golfer? Journal of Applied Sport Psychology, 22, 51-64. doi: $10.1080 / 10413200903403216$

Jackson, R. C. (2003). Pre-performance routine consistency: temporal analysis of goal kicking in the Rugby Union World Cup. Journal of Sports Sciences, 21, 803-814. doi: $10.1080 / 0264041031000140301$

Lonsdale, C., \& Tam, J. T. M. (2008). On the temporal and behavioural consistency of preperformance routines: An intra-individual analysis of elite basketball players' free throw shooting accuracy. Journal of Sports Sciences, 26, 259-266. doi:

$10.1080 / 02640410701473962$

Milton, J., Solodkin, A., Hlustik, P., \& Small, S. L. (2007). The mind of expert motor performance is cool and focused. Neuroimage, 35, 804-813. doi:

10.1016/j.neuroimage.2007.01.003

Singer, R. N. (2002). Preperformance state, routines, and automaticity: What does it take to realize expertise in self-paced events? Journal of Sport \& Exercise Psychology, 24, 359375.

Vickers, J. N., \& Williams, A. M. (2007). Performing under pressure: The effects of physiological arousal, cognitive anxiety, and gaze control in biathlon. Journal of Motor Behavior, 39, 381-394. doi: 10.3200/jmbr.39.5.381-394

Wulf, G., \& Prinz, W. (2001). Directing attention to movement effects enhances learning: A review. Psychonomic Bulletin \& Review, 8, 648-660. doi: 10.3758/bf03196201 